\title{
Second Virial Coefficient of Binary Polystyrene Mixtures in Cyclohexane below the Theta Temperature
}

\author{
Zhen ToNG and Yoshiyuki EINAGA \\ Department of Macromolecular Science, Osaka University, \\ Toyonaka, Osaka 560, Japan
}

(Received March 27, 1984)

\begin{abstract}
The second virial coefficient $A_{2}$ for binary polystyrene mixtures F1 (10000) +F7 (74000) and F4 (43600) + F40 (491000) in cyclohexane was determined by light scattering in the temperature range from $\theta$ down near the cloud point. Here, the figures in the parentheses indicate the weight-average molecular weights of the component "monodisperse" samples. It was found that $A_{2}$ did not depend on either the molecular weights of the component polymers or the composition of the mixture but varied only with temperature.

KEY WORDS Second Virial Coefficient / Polystyrene / Cyclohexane / Light

Scattering /
\end{abstract}

In our recent work, ${ }^{1}$ it was found that the second virial coefficient $A_{2}$ of "monodisperse". (i.e., very narrow-distribution) polystyrene in cyclohexane did not depend on the polymer molecular weight at temperatures below $\theta$, the theta temperature for the system. This finding immediately led us to the expectation that $A_{2}$ of polydisperse polystyrene in cyclohexane in the same temperature range may not depend on the average molecular weights or the molecular weight distribution of the sample. Thus, we carried out a light scattering determination of $A_{2}$ for binary mixtures of "monodisperse" polystyrenes in cyclohexane at temperatures from $\theta$ down to about $1^{\circ} \mathrm{C}$ above the cloud point. This paper presents the experimental data obtained.

\section{EXPERIMENTAL}

\section{Materials}

Four "monodisperse" polystyrene samples F1, F4, F40, and F7, the first three supplied by Toyo Soda Co., were subjected to precipitation and/or column fractionation. GPC measurements showed that the fractionated samples had $M_{w} / M_{n}$ ratios $\left(M_{w}\right.$ and $M_{n}$ are the weight-average and number-average molecular weights) not greater than 1.03, and hence these samples were regarded as essentially monodisperse in molecular weight.

The $M_{w}$ values for samples F1, F4, and F40, determined by light scattering in benzene at $25^{\circ} \mathrm{C}$, were 10000,43600 , and 491000 , respectively, and that for sample F7 by lowangle light scattering GPC was 74000 .

Solvent cyclohexane (Nakarai Chemical Co.) was freshly distilled over sodium before each light scattering experiment.

\section{Preparation of Solutions}

Binary mixtures F1 + F7 and F4 + F40 were used as test samples. The code and composition of each sample are shown in Table I, where $\xi$ denotes the weight fraction of the lower molecular weight component in the mixture.

Complete mixing of polymers was ensured by dissolving their mixture in benzene and then allowing the solvent to evaporate completely under vacuum. Test solutions were prepared by the procedure described previ- 
Table I. Composition and weight-average molecular weights of the binary polystyrene mixtures

\begin{tabular}{rllrr}
\hline \multicolumn{2}{c}{ Mixture } & \multicolumn{1}{c}{$\xi$} & $M_{\text {wcalcd }}$ & $M_{\text {wobsd }}$ \\
\hline F1+F7 & F7 & 0 & - & 74000 \\
B25 & 0.250 & 57800 & 59700 \\
B50 & 0.500 & 42000 & 41900 \\
B75 & 0.750 & 26000 & 26300 \\
F1 & 1 & - & 10000 \\
F4+F40 F40 & 0 & - & 491000 \\
S25 & 0.250 & 379000 & 379000 \\
S50 & 0.500 & 267000 & 288000 \\
S99 & 0.990 & 48100 & 49300 \\
F4 & 1 & - & 43600 \\
\hline
\end{tabular}

ously. ${ }^{1}$

The total polymer volume fraction $\phi$ was used as the concentration variable and defined by

$$
\phi=\left[1+\left(v_{0} / v_{\mathrm{p}}\right)\left(w^{-1}-1\right)\right]^{-1}
$$

where $w$ is the total polymer weight fraction, and $v_{0}$ and $v_{\mathrm{p}}$, the specific volumes of the solvent and the polymer, respectively. The values of $1.2923 \mathrm{~cm}^{3} \mathrm{~g}^{-1}$ and $0.9343 \mathrm{~cm}^{3} \mathrm{~g}^{-1}$, referring to $25^{\circ} \mathrm{C}$, were used for $v_{0}$ and $v_{\mathrm{p}}$, respectively, at any temperature studied.

\section{Light Scattering Measurements}

Scattering intensities from the solvent and solutions were measured at angles between 30 to $150^{\circ}$, using a Fica 50 photometer with vertically polarized incident light of wavelength $546.1 \mathrm{~nm}$. The apparatus was calibrated with benzene as the standard liquid. The temperatures studied ranged from the $\theta$ temperature $\left(34.5^{\circ} \mathrm{C}\right)$ to about $1^{\circ} \mathrm{C}$ above the cloud point of the test solution. Temperature fluctuation was less than $\pm 0.05^{\circ} \mathrm{C}$ during each measurement.

For a quasibinary polymer solution, the excess Rayleigh ratio $\Delta R_{0}$ at zero scattering angle is related to the derivatives $\mu_{i j}$ of the chemical potential $\mu_{i}$ of the solute component $i$ with respect to molality $m_{j}$ of the solute component $j$ by $^{2}$

$$
\Delta R_{0}=K_{\phi} R T \frac{\sum_{i} \sum_{j} \Delta_{i j}}{|\mu|}
$$

Here, $|\mu|$ is the determinant whose elements are $\mu_{i j}, \Delta_{i j}$ the cofactor for the element $\mu_{i j}$, and $R T$ has the usual meaning. The optical factor $K_{\phi}$ is given by

$$
K_{\phi}=\frac{4 \pi^{2} n^{2}}{N_{\mathrm{A}} \lambda_{0}{ }^{4}} V_{0}\left(\frac{\partial n}{\partial \phi}\right)^{2}
$$

where $n$ is the refractive index of the solution, $V_{0}$ the molar volume of the solvent, $N_{\mathrm{A}}$ the Avogadro constant, and $\partial n / \partial \phi$ the refractive index increment on the $\phi$ basis. Values of $\partial n / \partial \phi$ at various temperatures and concentrations were claculated from Scholte's equation $^{3,4}$ as described in the previous paper. ${ }^{1}$

\section{RESULTS AND DISCUSSION}

\section{Angular Dependence of $\Delta R_{\theta}$}

Figures 1a and 1b show $K_{\phi} / \Delta R_{\theta} v s \cdot \sin ^{2}(\theta / 2)$ plots for samples S25 and B75 at the indicated concentrations, where $\Delta R_{\theta}$ is the excess Rayleigh ratio at an angle of $\theta$. The data points for S25 at different temperatures are accurately fitted by straight lines, permitting $K_{\phi} / \Delta R_{0}$ to be evaluated by extrapolation to $\theta=0$. The data points for B75 at temperatures higher than $25^{\circ} \mathrm{C}$ also follow straight lines but those at lower temperatures markedly deviate downward from a linear relation as $\theta$ approaches $0^{\circ}$. The latter behavior was observed only in F1 + F7 mixtures at $\phi$ lower than 0.05 , and may be attributed to microphase separation in the solution. The $K_{\phi} / \Delta R_{0}$ values for those mixtures were estimated by extrapolating high angle data as illustrated by the dashed lines in Figure 1b.

\section{Molecular Weight $M_{w}$}

Figure 2 depicts the concentration dependence of $\phi K_{\phi} / \Delta R_{0}$ for samples B75 and S25. It can be seen that $\phi K_{\phi} / \Delta R_{0}$ at $34.5^{\circ} \mathrm{C}$ (the $\theta$ temperature) is almost constant at low $\phi$ and 
$A_{2}$ below $\theta$ for Ternary Solutions

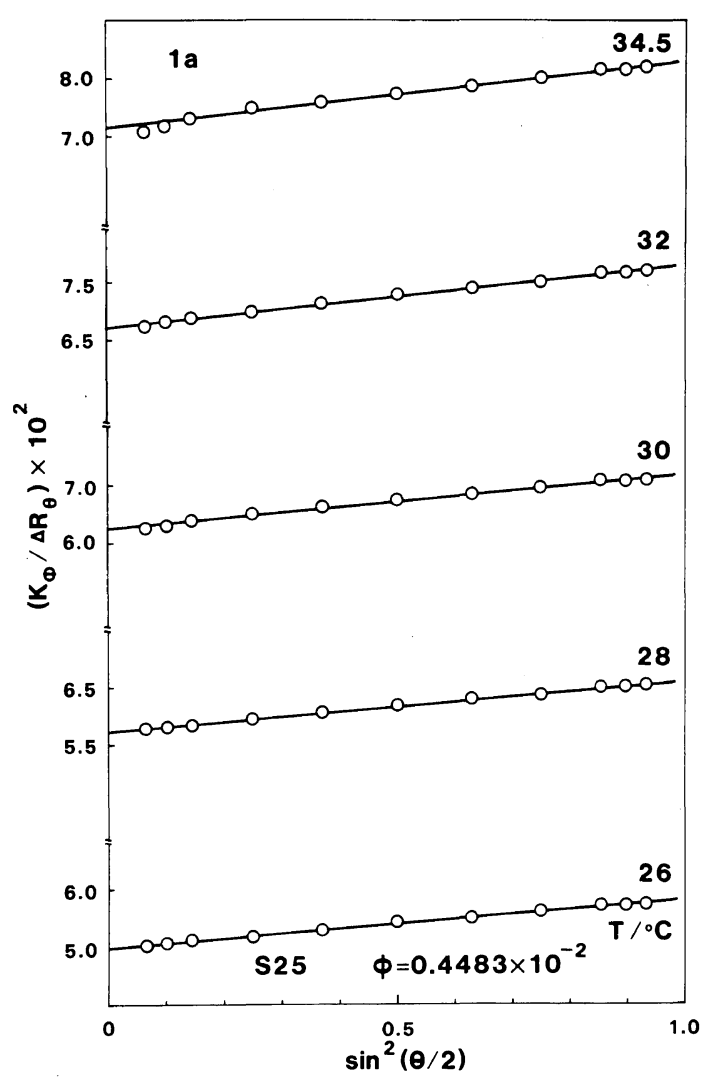

Figure 1a. Angular dependence of $K_{\phi} / \Delta R_{\theta}$ for the sample $\mathrm{S} 25$ at the temperatures indicated.

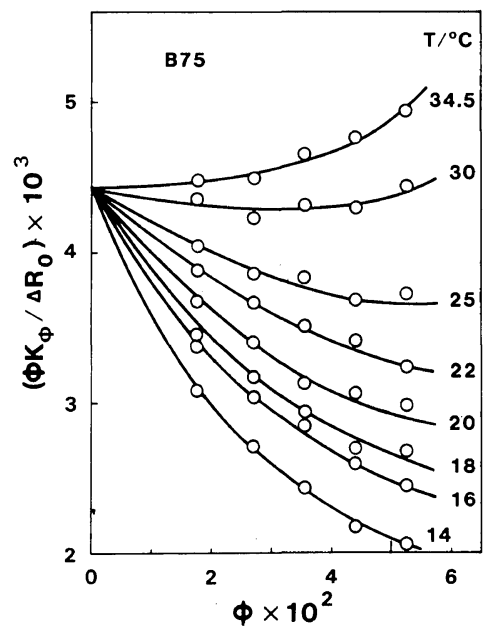

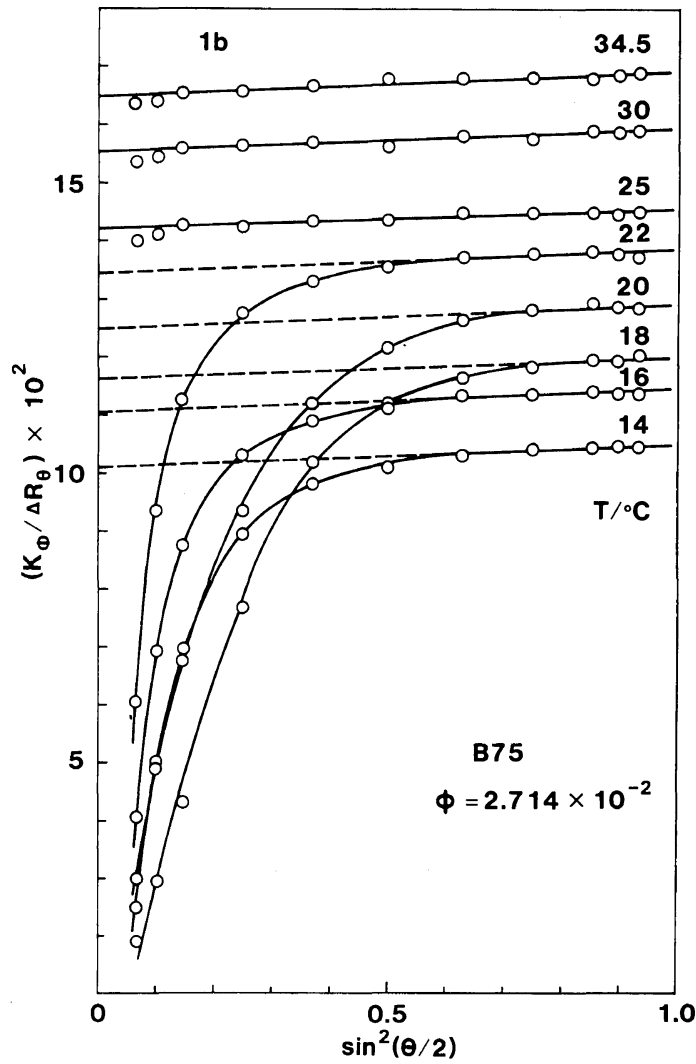

Figure 1b. Angular dependence of $K_{\phi} / \Delta R_{\theta}$ for the sample B75 at the temperatures indicated.

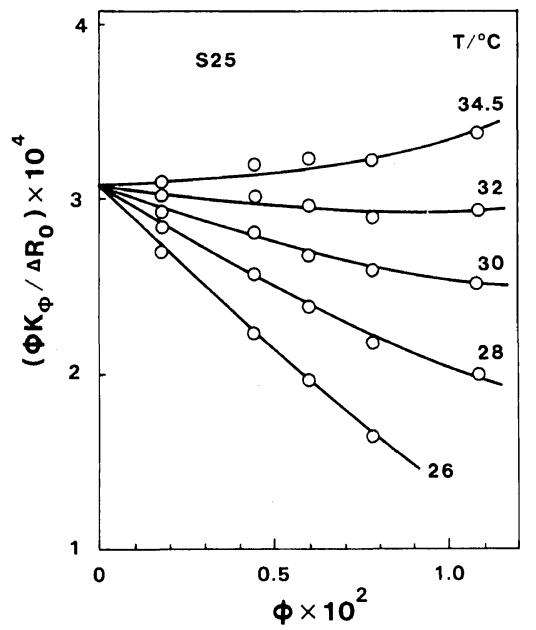

Figure 2. Plots of $\phi K_{\phi} / \Delta R_{0} v s$. $\phi$ for the samples $\mathrm{B} 75$ and $\mathrm{S} 25$ at the temperatures indicated. 
begins to increase at a relatively high $\phi$, indicating $A_{2}$ to be essentially zero at this temperature. The curves fitting the data points at lower temperatures have negative initial slopes and tend to bend upward at higher $\phi$, giving negative $A_{2}$ and higher virial coefficients which cannot be neglected.

For each mixture, the curves at different temperatures could all be extrapolated to the same ordinate intercept, which equals the inverse of the weight-average relative chain length $P_{w}$ for the mixture. The weight-average molecular weight $M_{w}$ was calculated from $P_{w}$ by

$$
M_{w}=\left(V_{0} / v_{\mathrm{p}}\right) P_{w}
$$

The values of $M_{\text {wobsd }}$ in Table I show $M_{w}$ so obtained for all the mixtures studied. These are in close agreement with the values $\left(M_{w c a l c d}\right)$ calculated from $M_{w}$ of the components and $\xi$ of the mixture, indicating that no intermolecular association occurred down to a temperature close to the cloud point when the solution was very dilute.

\section{Second Virial Coefficient $A_{2}$}

We define $Z$ by

$$
Z \equiv\left[1 / \phi P_{w}+1 /(1-\phi)-K_{\phi} / \Delta R_{0}\right] / 2
$$

which contains only experimentally measurable quantities. The general theory of light scattering ${ }^{2}$ shows that $K_{\phi} / \Delta R_{0}$ can be represented by

$$
K_{\phi} / \Delta R_{0}=1 / \phi P_{w}+2\left(V_{0} / v_{\mathrm{p}}{ }^{2}\right) A_{2}+0(\phi)
$$

Substitution of eq 6 into eq 5 gives the second virial coefficient $A_{2}$ in terms of $Z_{0}$ as

$$
A_{2}=\left(v_{\mathrm{p}}{ }^{2} / V_{0}\right)\left(1 / 2-Z_{0}\right)
$$

Here, $Z_{0}$ is the value of $Z$ at zero concentration. Figure 3 shows the $\phi$ dependence of $Z$ for the six polystyrene mixtures studied. The data points at each temperature follow a straight line or a curve somewhat bent upward and allow $Z_{0}$ to be evaluated accurately.

Figure 4 shows the $\xi$ dependence of $-A_{2}$ at
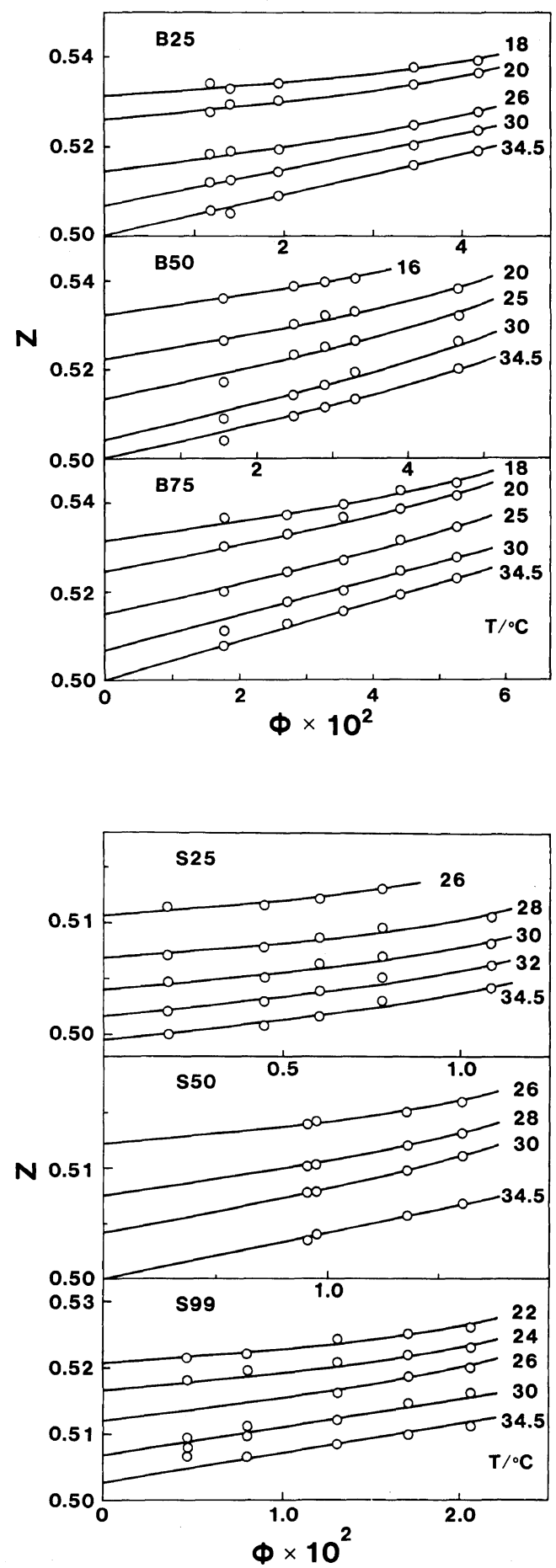

Figure 3. Concentration dependence of $Z$ for all the samples at the temperatures indicated; see eq 5 for the definition of $Z$. 


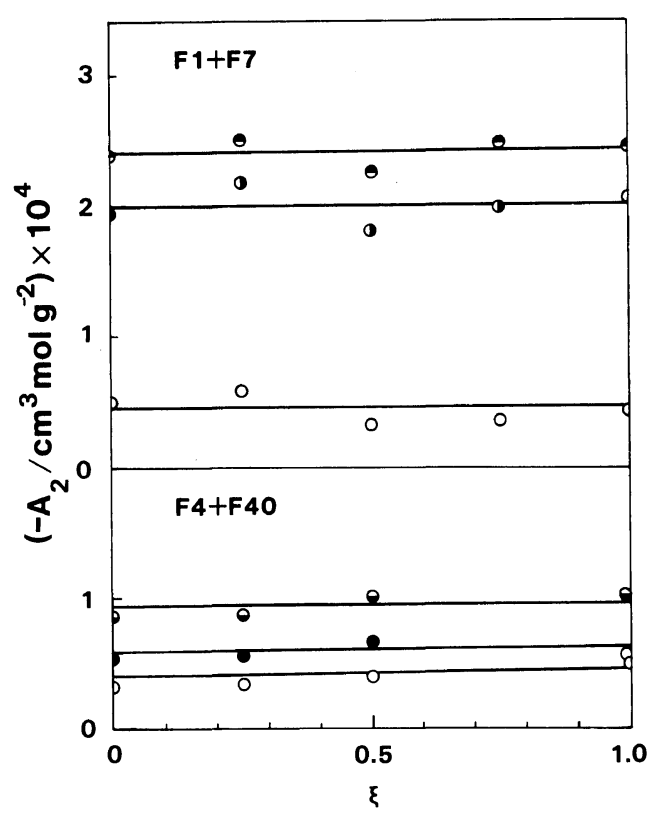

Figure 4. Plots of $-A_{2} v s$. $\xi$ for the mixtures $\mathrm{F} 1+\mathrm{F} 7$ and F4+F40. Temperatures are as follows: $\bigcirc, 30^{\circ} \mathrm{C} ; \boldsymbol{O}$, $28^{\circ} \mathrm{C} ; \ominus, 26^{\circ} \mathrm{C} ; \bigcirc, 20^{\circ} \mathrm{C} ; \ominus, 18^{\circ} \mathrm{C}$. Solid lines represent the mean values of $-A_{2}$ for the components of the mixtures. fixed temperatures below $\theta$ for two series of binary mixtures. Though the data points are somewhat scattered, those at each temperature can be fitted by a horizontal line representing the mean value of $-A_{2}$ for the components of the mixture. Thus, it may be concluded that $A_{2}$ for our polystyrene mixtures in cyclohexane below $\theta$ is essentially independent of $\xi$, i.e., the heterogeneity of the sample.

The data points in Figure 5 give $-A_{2}$ for all mixtures studied as a function of $T^{-1}$, where $T$ is the absolute temperature. They scatter around the solid line fitting the $A_{2}$ data previously obtained for "monodisperse" polystyrene in cyclohexane. This indicates that the molecular weights and mixing ratio of the component polystyrene samples virtually have no effect on $A_{2}$ in cyclohexane below $\theta$. Whether this is valid or not for a polystyrene mixture containing more than two components is left for future investigation.

Acknowledgements. We express our appre-

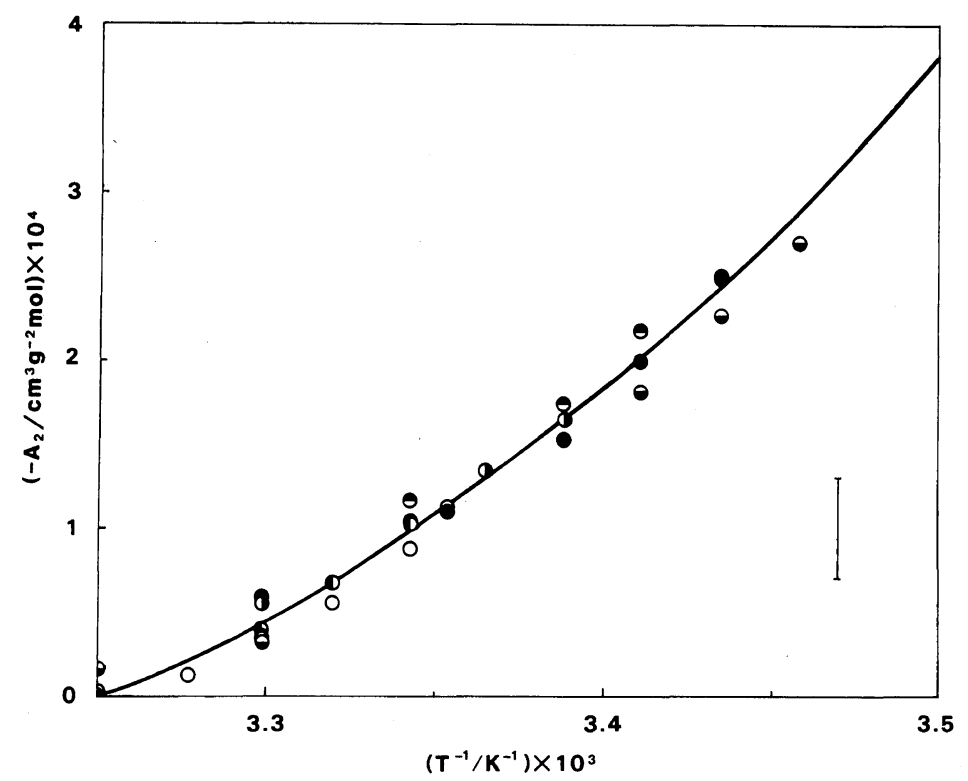

Figure 5. $-A_{2}$ plotted against $T^{-1}$ for the following samples: $\bigcirc, \mathrm{S} 25 ; \boldsymbol{D}, \mathrm{S} 50 ; \boldsymbol{\top}, \mathrm{S} 99 ; \ominus, \mathrm{B} 25 ; \ominus, \mathrm{B} 50$; , B75. Solid line represents the data obtained for monodisperse polystyrenes. The probable experimental error is indicated by a vertical bar in the figure. 
ciation to Professor H. Fujita for his assistance in the preparation of this manuscript. Z. Tong acknowledges the support of the government of China, by which he could study at the Graduate School of Macromolecular Science, Osaka University, Japan.

\section{REFERENCES}

1. Z. Tong, S. Ohashi, Y. Einaga, and H. Fujita, Polym. J., 15, 835 (1983).

2. M. Kurata, "Thermodynamics of Polymer Solutions," translated from the Japanese by H. Fujita, Harwood Academic, New York, 1982.

3. Th. G. Scholte, J. Polym. Sci., A-2, 8, 841 (1970).

4. Th. G. Scholte, Eur. Polym. J., 6, 1063 (1970). 